\title{
Contribución al catálogo de flora cubana: endemismos de suelos derivados de ofiolitas
}

\author{
Antonio López Almirall ${ }^{1}$
}

Resumen: López Almirall, A. 2013. Contribución al catálogo de flora cubana: endemismos de suelos derivados de ofiolitas. Bot. Complut. 37 : 135-152.

Las regiones cubanas donde predominan las ofiolitas son conocidas por tener más endemismos vegetales que regiones aledañas. La riqueza conjunta de esas floras es tal que para entender los patrones de distribución de la flora cubana hay que conocer esos patrones en regiones ofiolíticas. Buenos indicadores de la riqueza total son los componentes endémicos. Con ese fin aquí se publica un esquema cartográfico de regiones cubanas donde predominan las rocas mencionadas y una lista de taxones endémicos cubanos allí colectados. En los territorios identificados se han reconocido 1603 taxones infra genéricos endémicos cubanos de 106 familias y 420 géneros. Del total, 741 son exclusivos de regiones ofiolíticas. La mayor parte de los endemismos mencionados son arbustos, seguidos por las hierbas. En los suelos derivados de ofiolitas predominan principalmente los taxones del grupo evolutivo norandino.

Palabras clave: Cuba, fitorregiones, flora, hábitos.

Abstract: López Almirall, A. 2013. Contribution to the Cuban flora catalogue: endemics in the ophiolites derivate soils. Bot. Complut. 37: 135-152.

The Cuban regions with predominance of ophiolites are known to have more endemics plants than bordering regions. The combined wealth of those floras is such that to understand the patterns of distribution of the Cuban flora is necessary to know those patterns in ofilitic regions. Good indicators of the total wealth are the endemic components. With that objective here is published a cartographic diagram of Cuban regions where it prevail the mentioned rocks, and a list of Cuban endemic taxa collected there. In the identified territories has been recognized 1603 infra generic endemic taxa of 106 families and 420 genus. Of those total, 741 are exclusive of ophiolitic regions. In the soils derivate from ophiolitics rocks mainly taxa from the North Andean evolutionary group are predominate.

Key words: Cube, phytogeographic regions, flora, habits.

\section{INTRODUCCIÓN}

Entre los botánicos cubanos se han usado los términos «limonitas» (León 1946) y «serpentinas» (Samek 1973) para referirse al conjunto de rocas llamadas de manera genérica ofiolitas por los geólogos (Iturralde Vinent 1996). Carabia (1945) asocia la riqueza de endemismos en la flora cubana a la complejidad del relieve. Samek (1973) y Borhidi (1996) conceden mucha importancia a la flora endémica acumulada en las ofiolitas. López (1998) demuestra que con la complejidad del relieve aumenta la riqueza en especies, y con esta última aumenta la riqueza en endemismos. El mismo autor menciona la aridez y el sustrato como segundo y tercer factores que favorecen la riqueza. Sin embargo Cuchillas del Toa, una región ofiolítica muy antigua (Formell 1989) de relieve complejo po- see la mayor diversidad vegetal total y endémica del país. Esa riqueza influye de significativamente sobre las regiones aledañas (López 2005). La causa de esa riqueza se debe buscar en la sinergia entre los factores que allí se reúnen, a los que ya hicimos mención.

Es indiscutible que las plantas que viven en las ofiolitas se caracterizan por su especialización a ese sustrato (Wittaker 1960; Borhidi 1988, 1996), y en Cuba son comunes esas formaciones geológicas (Formell 1989, Iturralde Vinent 1996). Por las razones expuestas, es imprescindible conocer estas flórulas para entender la flora cubana en su conjunto. Un primer paso es identificar los endemismos, cuya riqueza y composición se correlaciona con la totalidad de la flora (Cracraft 1985, López 1998). De ahí la propuesta de este trabajo, donde se pretende censar los endemismos infragenéricos cubanos colectados en las ofiolitas.

\footnotetext{
${ }^{1}$ Museo Nacional de Historia Natural de Cuba, Obispo 61, Plaza de Armas, La Habana Vieja, La Habana 10100, Cuba. cycas@mnhnc.inf.cu Recibido: 26 septiembre 2012. Aceptado: 4 marzo 2013.
} 


\section{MATERIALES Y MÉTODOS}

Un inconveniente al estimar la distribución de los taxones son las indefiniciones territoriales de las regionalizaciones fitogeográficas más usadas en Cuba (Samek 1973, Borhidi \& Muñiz 1986). Para paliar esto, se hicieron modificaciones a López (2005), que es una versión enmendada de Samek (1973). Con ello se busca un acercamiento a criterios de geografía física, donde los límites de las unidades territoriales están definidos. La base cartográfica usada es la regionalización geomorfológica de Acevedo (1989). Los nombres asignados a los distritos fitogeográficos se acercan en lo posible a los términos usados por el mencionado autor y los que aparecen en el mapa 1:250000 del Instituto Cubano de Geodesia y Cartografía (Fig. 1). El uso del relieve se justifica por la importancia de esta variable en la distribución de la flora cubana, como señalamos. En esa regionalización se seleccionaron los distritos con predominancia de ofiolitas, de acuerdo con los criterios de Howard (1973), Samek (1973), Berazaín (1986), Borhidi (1996), Formell (1989), Capote et al. (1989) e Iturralde Vinent (1996). Los distritos escogidos fueron: Cajálbana (9); Colinas de Habana-Limonar (13), Motembo (15) y Santa Clara (18) en el Cuba Occidental; Camagüey (21) y Holguín (24), en Cuba Central, y Sierra de Nipe (32), Sierra de Cristal (33) y Moa (35) en Cuba Oriental (Fig. 1).

A continuación se segregaron en la base de datos de endemismos vegetales del Museo Nacional de Historia Natural todos los registros de taxones presentes en los distritos mencionados. En esta base de datos están registrados los ejemplares de endemismos cubanos depositados en el Herbario Nacional (HAC), Museo Nacional de Historia Natural de Cuba (MNHN), Smith- sonian Institution (US) y Jardín Botánico de Montreal (MTJB). La base recoge también los ejemplares revisados por autores que describieron especies a partir del Suplemento de la flora de $\mathrm{Cu}$ ba (Liogier 1974). La validez de los taxones en la base de datos del Museo se rectificó entre los años 2010 y 2011 con ayuda «on line» de las bases de datos: Catalogue of Life 2011 (www.catalogueoflife.org/annual-checklist), Tropicos (www.tropicos.org), The Plant List (www.theplantlist.org) y The International Plant Name Index (www.ipni.org). Los resultados de esta revisión se contrastaron con la lista de Acevedo Rodríguez \& Strong (2012). En los casos de discrepancia de la lista con las bases de datos mencionadas, aceptamos la segunda fuente de información. En esa revisión se evidenció una reducción sensible del número de endemismos infragenéricos totales cubanos hasta cifras similares a las estimadas por Alain (1958). Las principales causas de esa reducción fueron: 1. la ampliación del área de distribución de muchos taxones a otras regiones del Caribe. 2. taxones que pasaron a la sinonimia de otros, endémicos o no.

Una vez segregados los taxones, se dividieron en tres grupos de distribución: 1. endemismos totales, 2. exclusivos de las ofiolitas y 3. no exclusivos. En el primer grupo están todos los taxones escogidos, en el segundo aquellos que solo se colectaron en los distritos ofiolíticos y en el tercero, los que viven también fuera de esos distritos. Esa división pone en evidencia los patrones generales de distribución de los taxones involucrados, su estructura taxonómica, composición por hábitos, y por centros evolutivos neotropicales. Eso permitió una valoración global sobre la intensidad de la especiación ocurrida en esos territorios, y el grado de aridez de las formaciones vegetales predominantes en cada distrito. Para los centros evolutivos se

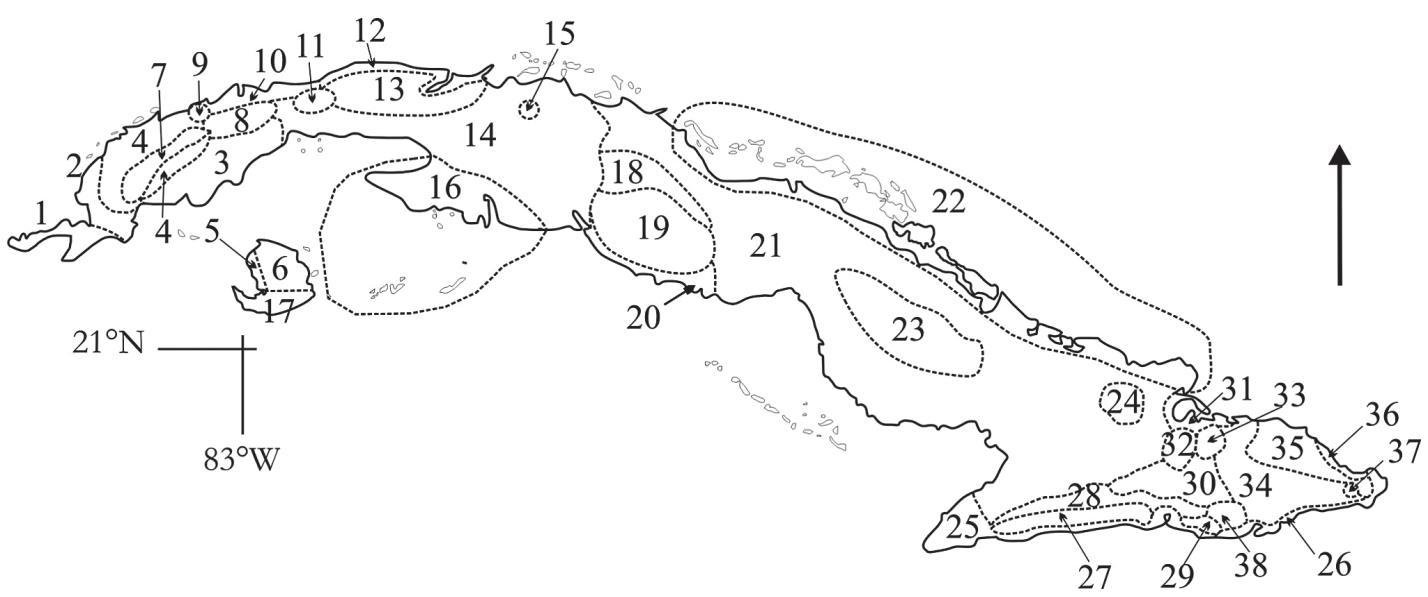

Fig. 1-Esquema cartográfico con las regiones fitogeográficas de Cuba. 1: Guanahacabibes. 2: Guane. 3: Pinar del Río. 4: Pizarras. 5: Los Indios-Siguanea. 6: Centro de Pinos. 7: Mogotes. 8: Sierra del Rosario. 9: Cajálbana. 10: Bahía Honda Cabañas. 11: Anafe. 12: Costa Norte Habana-Matanzas. 13: Alturas Habana-Limonar. 14: Llanuras Centro-Occidentales. 15: Motembo. 16: Zapata. 17: Sur Isla de la Juventud. 18: Santa Clara. 19: Guamuhaya. 20: Cienfuegos-Trinidad. 21: Camagüey. 22: Llanuras Centro-Orientales. 23: Costa Norte Centro-Oriental. 24: Holguín. 25: Media Luna-Cabo Cruz-Baconao. 26: Maisí Guantánamo. 27: Cordillera del Turquino. 28: Promontorios de Sierra Maestra. 29: Gran Piedra. 30: Valles Centrales. 31: Bahía de Nipe. 32: Sierra de Nipe. 33: Sierra Cristal. 34: Meseta del Guaso. 35: Cuchillas del Toa. 36: Baracoa. 37: Sierra de Puriales. 38: Colinas de Oriente. 
usaron los neotropicales de Gentry (1982), aunque con modificaciones necesarias después de treinta años. La primera es reconocer un centro evolutivo chaqueño. Para ello se tomó como base la particularización que el autor hace sobre la composición y hábitos de los taxones predominante en los cerrados, y la distribución de muchos presentes en Cuba. Las Antillas Mayores comparten un número significativo de géneros con esas formaciones vegetales, entre los cuales sobresale Copernicia, que está desde Cuba y la Española hasta el Chaco. El clima de los «cerrados» es diferente al que tienen los centros evolutivos reconocidos por el autor, donde predominan pluvisilvas. El clima de los cerrados coincide con el de sabanas aunque allí predominan los matorrales tropicales (Goodland \& Ferri 1979; Raven et al. 1994, 1992; Raven \& Jhonson 2002).

Otra modificación es la definición biogeográfica de esos centros evolutivos adaptándolos a Morrone (2001). De esa forma el centro evolutivo amazónico comprende la subregión Amazónica salvo las provincias biogeográficas de Guyana y Guyana Húmeda, que conforman el centro evolutivo de Guyana. El centro llamado Andes Boreales corresponde a las provincias Chocó, Cauca y Magdalena. El Chaqueño, se identifica con la región del mismo nombre, donde hay una provincia llamada Cerrado. Los Andes australes de Gentry (1982) con las porciones neotropicales de los Andes como: Páramo Andino y el Desierto Peruano. Hay dos centros de Gentry (1982) sin ubicación geográfica: las regiones áridas y el indefinido. En el primero incluye un grupo de familias presente en las regiones áridas de las Américas, derivada de Laurasia en el norte y Gondwana en el sur. Las familias mejor conocidas de este grupo son: Cactaceae y Agavaceae, que dan unidad fitogeográfica los desiertos de América. En el grupo indefinido están familias cuyo centro evolutivo no pudo definir el autor. A esos grupos, se añadió el grupo indeterminado, con familias que el autor de esta publicación no pudo poner en ninguno de los otros.

La aridez biológica de la vegetación en los territorios ofiolíticos se determinó dividiendo el número de endemismos totales perteneciente a familias del centro evolutivo chaqueño entre los pertenecientes a familias norandinas Ferrás et al. (1999). La intensidad de especiación es el porcentaje de endemismos exclusivos de las ofiolitas en relación con el total de endemismos cubanos presentes. El cálculo de especiación se basa en una regla general, presente de forma más o menos explícita en casi todas las publicaciones biogeográficas. Esa regla estipula que difícilmente el centro de origen de una especie está allí donde la encontramos (Espinosa Organista \& Llorente Bousquet 1993). En contraposición a esta regla, Gentry (1990) afirma que las especies de distribución limitada se originaron en algún lugar del territorio que ocupan.

Cuba tiene algo más de $120.000 \mathrm{~km}^{2}$, y son pocos los endemismos presentes en toda el Archipiélago. Por tanto, todos los endemismos exclusivos de las ofiolitas ocupan territorios inferiores a los $100.000 \mathrm{~km}^{2}$. Eso significa que todos tienen distribución limitada en un sentido estricto, y por tanto cumplen la condición exigida por Gentry (1990). Para completar, se incluye la lista de los endemismos infragenéricos censados en las ofiolitas cubanas (Anexo 1).

\section{RESULTADOS Y DISCUSIÓN}

Acevedo Rodríguez \& Strong (2012) reconocen para Cuba 5778 taxones nativos, sin especificar su nivel, por lo que asumimos que se refieren a taxones infragenéricos. De acuerdo con estos autores, en Cuba hay 2980 plantas con semillas endémicas, para un $51 \%$. En la base de datos del Museo Nacional de Historia Natural después de su actualización, se reconocen 2787 taxones, lo cual significa $48 \%$ del total de taxones nativos señalados por Acevedo Rodríguez \& Strong (2012). Ese porcentaje se eleva si se restan los endemismos que han sido invalidados de acuerdo con las bases de datos «on line» consultadas.

En suelos cubanos derivados de ofiolitas se han colectado 1603 taxones infra genéricos endémicos (Anexo 1), el 58\% de los endemismos conocidos. Estos taxones están incluidos en 106 familias y 420 géneros. De los 1603 taxones hay 741 exclusivos de estos suelos. Lo cual indica una intensidad de especiación cercana a la mitad de los taxones.

Aunque los endemismos cubanos presentes en las ofiolitas, se distribuyen en 106 familias, diez de ellas acumulan casi el 55\% de los taxones infra genéricos. Esas familias, con treinta o más endemismos cubanos colectadas en esos lugares son, en orden decreciente: Rubiaceae, Myrtaceae, Euphorbiaceae, Asteraceae, Melastomataceae, Leguminosae, Orchidaceae, Arecaceae, Poaceae y Verbenaceae. Otro aspecto curioso es la correlación tan estrecha entre la riqueza en endemismos totales y exclusivos de las familias (Fig. 2).

En los géneros el patrón de alguna manera se repite. El 9\% de los géneros con endemismos totales acumula $44 \%$ de los taxones, y $7 \%$ de los que tienen endemismos exclusivos acumula $37 \%$ de los taxones. Con veinte 0 más endemismos totales se distinguen: Eugenia, Calyptran-

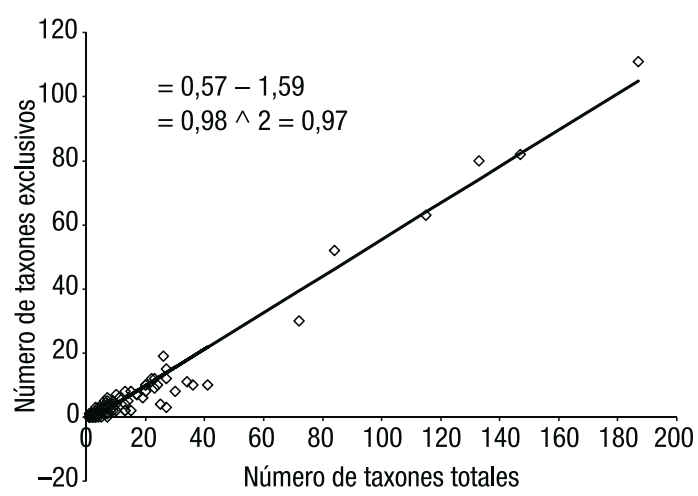

Fig. 2- Correlación entre taxones totales y exclusivos por familia. 
thes, Rondeletia, Phyllanthus, Psychotria, Buxus, Leucocroton, Ossaea, Coccoloba, Croton y Gesneria. Los géneros que más han especiado, con más de quince taxones exclusivos en las ofiolitas son: Calyptranthes, Eugenia, Rondeletia, Phyllanthus, Buxus, Leucocroton, Schmidtottia, Ossaea y Croton. El coeficiente de correlación entre endemismos cubanos totales y exclusivos por género, supera ligeramente el valor de 0,9 .

Las floras endémicas cubanas, sobre suelos derivados de ofiolitas, al igual que la mayoría de las floras locales del Archipiélago, se distinguen por el predomino de arbustos. Ese predominio es alrededor de las dos terceras partes de los endemismos totales y los no exclusivos. Entre los exclusivos el porcentaje de arbustos supera las tres cuartas partes. A diferencia de lo que ocurre en otras regiones de Cuba (López 1994, López et al. 1994), el segundo lugar en riqueza de endemismos lo ocupan las herbáceas, no los árboles. El porcentaje de trepadoras totales supera el $5 \%$, debido a que los entre taxones exclusivos constituyen el 8\%. La mayor intensidad de especiación corresponde a los arbustos, con $52 \%$ y la menor a las trepadoras, con $37 \%$. Las especies con otros hábitos están entre esos valores. En las tres categorías de endemismos predominan los componentes asociados al centro evolutivo norandino, seguido del chaqueño. El tercer lugar lo ocupan los componentes laurásicos y los asociados a los andes australes.

La aridez biológica en el conjunto de biotas sobre ofiolitas es de 0,74 este valor, inferior a la unidad, corresponde a regiones que sostienen formaciones vegetales con formaciones vegetales húmedas. Este valor medio probablemente está influido por la riqueza tan alta de las Cuchillas del Toa, donde hay montañas con precipitaciones anuales superiores a $2000 \mathrm{~mm}$. En las ofiolitas de regiones de relieve menos complejo, solo hay matorrales áridos. De ahí la afirmación de Borhidi (1996), quien asegura que la estructura de la vegetación sobre ofiolitas equivale a la de regiones con predominancia de calizas donde caen $500 \mathrm{~mm}$ anuales menos de lluvia.

\section{BIBLIOGRAFÍA}

AcEvedo, M. 1989. Regionalización geomorfológica. En: Nuevo Atlas Nacional de Cuba, Sección IV-4: 3. Instituto de Geografía, Academia de Ciencias de Cuba.

Acevedo Rodríguez, P. \& Strong, M. T. 2012. Catalogue of Seed Plants of the West Indies. Smithsonian Institution Scholary Press, Washingtong DC.

Alain, HnO. 1958. La flora de Cuba: sus principales características, su origen probable. 2. Rev. Soc. Cubana Bot. 15(4): 84-96.

BERAZAín, R. 1986. Algunos aspectos fitogeográficos de plantas serpentínicolas cubanas. Feddes Reper. 97: 49-58.

Borhidi, A. 1996. Phytogeography and vegetation ecology of Cuba. Akadémiai Kiado, Budapest.

BorHIDI, A. 1988. El efecto ecológico de la roca serpentina a la flora y la vegetación de Cuba. Acta Acta. Bot. Hung. 34(12): 123-174.

BorHiDI, A. \& MuÑIZ, O. 1986. The phytogeographic survey of Cuba. 2. Floristic relationships and phytogeographic subdivision. Acta. Bot. Hung. 32(1-2): 3-48.

Capote, R.; Ricardo, N.; García, E. E.; Vilamajó, D., \& URBINO, J. 1989. Vegetación actual. En Nuevo Atlas Nacional de Cuba, Sección X-1: 2-3. Instituto de Geografía, Academia de Ciencias de Cuba, La Habana.

Carabia, J. P. 1945. The vegetation of Sierra de Nipe, Cuba. Ecol. Monogr. 15(4): 321-341.

CRACRAFT, J. 1985. Biological diversification and it causes. Ann. Missouri Bot. Gard. 72: 794-822.

Espinosa Organista, D. \& Llorente Bousquet, J. 1993. Fundamentos de biogeografías filogenéticas. Facultad de Ciencias UNAM, México
Ferrás, H.; López, A.; Martel, A., \& SuÁrez, A. 1999. Relaciones de la diversidad en la flora endémica cubana con la vegetación y la aridez del clima. II. Estudio de las variables climáticas. Acta Bot. Cubana 36: 1-12.

Formell, F. 1989. Constitución geológica. En Nuevo Atlas Nacional de Cuba, Sección III-1: 2-3. Instituto de Geografía, Academia de Ciencias de Cuba, La Habana.

Gentry A. H. 1982. Neotropical floristic diversity: Phytogeographical connections between Central and South America. Pleistocene climatic fluctuations or an accident of the Andean orogeny? Ann. Missouri Bot. Gard. 69(30): 557-593.

Gentry, A. H. 1990. Evolutionary patterns in neotropical Bignoniaceae. Mem. NY. Bot. Gard. 55: 118-129.

Goodland, R. \& Ferri, M. G. 1979. Ecologia do cerrado. Livraria Itaitaia, Sao Paulo.

Howard, R. A. 1973. The vegetation of the Antilles. En: A. Graham (Ed.) Vegetation and vegetational history of Northern Latin America: 1-38. Elsevier Scientific Publication, Amsterdam.

ItURRALDE Vinent, M. 1996. Geología de las ofiolitas cubanas. En: M. Iturralde (Ed.) Ofiolitas y arcos volcánicos de $\mathrm{Cu}$ ba: 83-119. IUG/UNESCO, Miami.

León, Hno. 1946. Flora de Cuba. 1. Contr. Ocas. Mus. Hist. Nat. Colegio La Salle 8: 1-441.

Liogier, A. H. H. 1974. Flora de Cuba. Suplemento. Instituto Cubano del Libro, La Habana.

LóPEZ, A. 1994. El endemismo vegetal en Moa-Toa-Baracoa (Cuba Oriental). Fontqueria 39: 433-473.

LóPEZ,A. 1998. Origen probable de la flora cubana. En G. Halffter (Ed.) La diversidad biológica de Iberoamérica, 2. Volu- 
men especial. Acta Zoológica Mexicana, nueva Serie: 83108. Instituto de Ecología, Xalapa.

LóPEZ, A. 2005. Nueva perspectiva para la regionalización fitogeográfica de Cuba: definición de los sectores. En: J. Llorente Bousquets \& J. J. Morrone (Eds.) Regionalización biogeográfica en Iberoamérica y trópicos afines. Primeras Jornadas Biogeográficas de la Red Iberoamericana de Biogeografía y Entomología Sistemática: 417-428. Universidad Nacional Autónoma de México, México D.F.

LóPez, A.; Rodríguez, M. \& CÁRdenAs, A. 1994. El endemismo vegetal de la Cordillera del Turquino (Cuba Oriental). Fontqueria 39: 395-431.
Morrone, J. J. 2001. Biogeografia de América Latina y el Caribe. M \& T Manuales \& Tesis SEA, 3. Zaragoza.

Morrone, J. J.; Evert, R. I. \& Eichorn, S. E. 1992. Biología de las plantas. Reverté, Barcelona.

Morrone, J. J. \& Johnson, G. 2002. Biology, $6^{\text {th }}$ Ed. McGraw Hill, New York.

SAmeK, V. 1973. Regiones fitogeográficas de Cuba. Acad. Cienc. Cuba, ser. forest. 15: 1-63.

WitTAKER, R. H. 1960. Vegetation of the Siskiyou Mountains, Oregon and California. Ecol. Monogr. 30(3): 279-338.

Anexo 1. Taxones infragenéricos endémicos cubanos que han sido colectados en suelos derivados de ofiolitas.

${ }^{1}$ Taxones colectados únicamente en las ofiolitas

Abarema asplenifolia R. C. Barneby \& J. W. Grimes

A. nipensis R. C. Barneby \& J. W. Grimes

${ }^{1}$ Acacia belairioides Urb.

A. bucheri Vict.

A. daemon Ekman \& Urb.

Acalypha havanensis Müll. Arg.

${ }^{1}$ A. nana Griseb.

${ }^{1}$ Acianthera bissei G. M. Luer

${ }^{1}$ A. papulifolia G. M. Luer

A. rubroviridis Pridgeon \& M. W. Chase

Acidocroton acunae Borhidi \& O. Muñiz

${ }^{1}$ A. trichophyllus subsp. pilosulus Borhidi

Acinodendron obtusum Kuntze

Acrocomia crispa C. F. Baker

${ }^{1}$ Acrosynanthus lucidus Britton

${ }^{1} A$. ovatus Urb.

${ }^{1}$ A. parvifolius Britton ex Standl.

${ }^{1}$ A. revolutus Urb.

${ }^{1}$ A. trachyphyllus Standl.

Acunaeanthus tinifolius Borhidi

Adenoa cubensis Arbo

Agave brittoniana Trel. subsp. brittoniana

${ }^{1}$ A. cajalbanensis A. Álvarez

${ }^{1}$ A. jarucoensis A. Álvarez

A. offoyana De Smet ex Jacobi

${ }^{1}$ A. shaferi Trel.

A. tubulata Trel. subsp. tubulata

A. underwoodii Trel.

${ }^{1}$ Allophylus cristalensis Lippold

Alvaradoa arborescens Griseb.

${ }^{1}$ Amphiolanthus bryoides Griseb.

${ }^{1}$ Amyris stromatophylla subsp. moaensis Borhidi \& 0. Muñiz

${ }^{1}$ A. stromatophylla P. Wilson subsp. stromatophylla

A. stromatophylla subsp. yumuriensis Borhidi \& O. Muñiz

Andropogon reedii C. L. Hitchc. \& Ekman

A. virginicus var. graciliformis Léon

Angadenia cubensis Miers.

A. lindeniana Miers.

${ }^{1} A$. moaensis Lippold

A. sagraei Miers.

Annona bullata A. Rich.
${ }^{1}$ A. cristalensis Z. M. Fernández

${ }^{1}$ A. ekmanii R. E. Fries

A. moaensis Léon \& Alain

${ }^{1} A$. nipensis Alain

${ }^{1}$ A. oblongifolia R. E. Fries

Anthurium gymnopus Griseb.

A. venosum Griseb.

${ }^{1}$ Antillanthus almironcillo B. Nord.

${ }^{1}$ A. azulensis B. Nord.

${ }^{1} A$. biseriatus $\mathrm{B}$. Nord

${ }^{1}$ A. carinatus B. Nord.

${ }^{1} A$. cubensis B. Nord.

${ }^{1}$ A. ekmanii B. Nord

${ }^{1}$ A. eriocarphus B. Nord

${ }^{1}$ A. leucolepis B. Nord.

${ }^{1}$ A. moaensis B. Nord

${ }^{1} A$. moldenkei Greenm. ex Greenm.

${ }^{1} A$. pachylepis B. Nord

${ }^{1}$ A. pachypodus B. Nord

${ }^{1}$ A. shaferi B. Nord.

${ }^{1}$ A. subsquarrosus B. Nord

A. trichotomus B. Nord.

${ }^{1}$ Antirhea ferruginea $\mathrm{C}$. Wright ex Griseb.

Aralia rex J. Wen

Arceuthobium cubense Leiva \& Bisse

${ }^{1}$ Ardisia grisebachiana Alain

A. curtifolia C. L. Hitchc.

A. erecta C. L. Hitchc.

A. laevigata C. L. Hitchc. \& Ekman

${ }^{1}$ A. pradana Léon

Aristolochia glandulosa J. Kickx f.

A. lindeniana Duch. var. lindeniana

A. passifloraefolia A. Rich.

Arthrostylidium cubense Rupr.

A. fimbriatum Griseb.

A. urbanii Pilg.

Asketanthera calycosa R. E. Woodson

Aster burgessii Britton

${ }^{1}$ Auerodendron acunae Borhidi \& 0 . Muñiz

A. glaucescens Urb.

${ }^{1}$ Ayenia cajalbanensis Alain 
A. euphrasiaefolia subsp. ophiticola A. Rodr. A. velutina Urb.

Baccharis halimifolia Linn var. angustior DC.

${ }^{1} B$. nipensis Urb.

B. scoparioides Griseb.

B. shaferi Britton

${ }^{1}$ B. micromonnieria Borhidi

${ }^{1}$ B. minuta Borhidi \& 0 . Muñiz

Bactris cubensis Burret

Badiera cubensis Britton

${ }^{1} B$. virgata subsp. alternifolia $\mathrm{R}$. Rankin

B. virgata subsp. virgata

Banisteriopsis pauciflora C. B. Rob.

${ }^{1}$ Basiphyllaea carabiaiana Sosa \& M. A. Díaz

${ }^{1} B$. wrightii Nir

${ }^{1}$ Begonia bissei J. Sierra

B. libanensis Urb.

B. wrightiana A. DC.

Behaimia cubensis Griseb.

Berrya cubensis M. Gómez subsp. cubensis

Bisgoeppertia gracilis Kuntze

Bissea myrtifolia V. R. Fuentes

Bonania cubana subsp. microphylla Borhidi

${ }^{1}$ B. emarginata subsp. nipensis Borhidi

${ }^{1}$ B. emarginata subsp. suborbiculata Borhidi \& Urbino

Bonellia brevifolia B. Ståhl \& Kallersjo

B. shaferi B. Ståhl \& Kallersjo

${ }^{1}$ Bonnetia cubensis R. A. Howard

Bourreria badia O. E. Schulz

B. homalophylla O. E. Schulz

B. moaensis Britton

B. pauciflora O. E. Schulz

B. rotata I. M. Johnst.

B. setoso-hispida O. E. Schulz

B. virgata var. cuneifolia Gottschling

Broughtonia cubensis Cogn.

Brunfelsia acunae Hadac

B. cestroides A. Rich.

B. grisebachii Amsh.

B. linearis Ekman

${ }^{1}$ B. pluriflora Urb.

B. purpurea Griseb.

${ }^{1}$ B. shaferi Britton \& P. Wilson

B. sinuata A. Rich.

Brya chrysogonii Léon \& Alain

${ }^{1} B$. hirsuta Borhidi

B. microphylla Bisse

B. subinermis Léon \& Alain

Bucida ophiticola Bisse

Bulbostylis setacea Svenson

Bunchosia emarginata Regel.

B. linearifolia P. Wilson

Buxus acuminata Müll. Arg.

${ }^{1}$ B. acunae Borhidi \& 0 . Muñiz

${ }^{1} B$. aneura Urb.

${ }^{1}$ B. baracoensis Borhidi \& 0. Muñiz

B. ekmanii Urb.

${ }^{1} B$. excisa Urb.

B. flaviramea Mathou

${ }^{1}$ B. foliosa Urb.
B. gonoclada Müll. Arg.

${ }^{1} B$. heterophylla Urb.

${ }^{1} B$. historica Borhidi \& O. Muñiz

${ }^{1}$ B. imbricata Urb.

${ }^{1}$ B. marginalis Urb.

${ }^{1} B$. moana Alain

B. muelleriana Urb.

${ }^{1}$ B. obovata Urb.

${ }^{1}$ B. olivacea Urb.

${ }^{1}$ B. pilosula Urb.

$B$. retusa Müll. Arg.

${ }^{1}$ B. revoluta Mathou

${ }^{1} B$. rheedioides Urb.

${ }^{1}$ B. rotundifolia Mathou

${ }^{1} B$. serpentinicola $\mathrm{E}$. Köhler

${ }^{1} B$. shaferi Urb.

${ }^{1} B$. vaccinioides Urb.

B. wrightii Müll. Arg.

Byrsonima biflora Griseb.

${ }^{1} B$. bucherae C. Moldenke

${ }^{1}$ B. minutifolia Alain

'B. moensis Acuña \& Roig

${ }^{1}$ B. motembensis Britton \& Small

B. orientensis Bisse

Caesalpinia bahamensis subsp. rugeliana Borhidi

C. myabensis var. hermeliae A. Barreto

C. myabensis var. subglauca A. Barreto

${ }^{1} C$. nipensis Urb.

C. pinnata subsp. oblongifolia A. Barreto \& Beyra

C. pinnata subsp. savannarum Borhidi \& 0. Muñiz

C. wrightiana Urb.

${ }^{1}$ Calliandra enervis Urb.

C. pauciflora subsp. pauciflora

Callicarpa areolata Urb.

C. crassinervis Urb.

C. cubensis Urb.

C. cuneifolia Britton \& P. Wilson

${ }^{1}$ C. ekmanii C. Moldenke

C. fulva A. Rich. var. fulva

C. fulva var. glabrescens C. Moldenke

C. lancifolia Merrill.

${ }^{1}$ C. leonis C. Moldenke

${ }^{1}$ C. moana Borhidi \& O. Muñiz

${ }^{1}$ C. nipensis Britton \& P. Wilson

C. oblanceolata Urb.

C. resinosa C. Wright \& C. Moldenke

${ }^{1}$ C. revoluta $\mathrm{C}$. Moldenke

C. toaensis Borhidi \& O. Muñiz

C. wrightii Britton \& P. Wilson

Calophyllum pinetorum Bisse

C. rivulare Bisse

C. utile Bisse

${ }^{1}$ Calycogonium acunanum Borhidi \& 0 . Muñiz

${ }^{1} C$. angulatum Griseb.

${ }^{1}$ C. clidemioides Griseb.

${ }^{1}$ C. cocoense Alain

${ }^{1}$ C. ellipticum C. Wright ex Sauvalle

${ }^{1} C$. grisebachii var. cristalense Borhidi

${ }^{1}$ C. grisebachii Triana var. grisebachii

C. lindenianum Naud. 
${ }^{1}$ C. moanum Borhidi

${ }^{1}$ C. perezii Alain

${ }^{1}$ C. revolutum Alain

${ }^{1}$ C. rosmarinifolium subsp. brachyphyllum Borhidi \& 0. Muñiz

${ }^{1}$ C. rosmarinifolium subsp. rosmarinifolium

${ }^{1}$ C. rubens Borhidi

C. susannae Borhidi

${ }^{1}$ Calycolpus cristalense Bisse

${ }^{1}$ Calyptranthes acunae Borhidi \& 0. Muñiz

${ }^{1}$ C. albicans Borhidi

${ }^{1}$ C. apoda Urb.

${ }^{1}$ C. baracoensis Borhidi

${ }^{1}$ C. bialata Urb.

${ }^{1}$ C. calyptrata Griseb.

${ }^{1}$ C. canapuensis Urb.

C. capitulata C. Wright ex Sauvalle var. capitulata

C. capitulata var. caroli Borhidi \& O. Muñiz

${ }^{1}$ C. cardiophylla Urb.

${ }^{1}$ C. compressa Urb.

${ }^{1}$ C. cristalensis Borhidi

${ }^{1}$ C. cuprea 0. Berg

C. decandra Griseb.

${ }^{1}$ C. elongata Urb.

C. enneantha $\mathrm{C}$. Wright

C. ermitensis Borhidi

${ }^{1}$ C. exasperata Borhidi

${ }^{1} \mathrm{C}$. heterochroa Urb.

C. leptoclada Urb.

${ }^{1}$ C. levisensis Bisse \& A. Rodr.

${ }^{1} \mathrm{C}$. linearis Alain

C. maestrensis Urb.

${ }^{1}$ C. mayarensis Borhidi

C. micrantha C. Wright

${ }^{1}$ C. minutiflora Borhidi

${ }^{1}$ C. moaensis Alain

${ }^{1}$ C. monocarpa Urb.

${ }^{1}$ C. munizii Borhidi

${ }^{1}$ C. nipensis Borhidi \& O. Muñiz

${ }^{1}$ C. oblanceolata Urb.

${ }^{1}$ C. oblongifolia R. A. Howard

${ }^{1}$ C. oligantha Urb.

${ }^{1}$ C. paradoxa Urb.

${ }^{1}$ C. pocsiana Borhidi

C. polysticta Urb.

${ }^{1}$ C. pseudoapoda Bisse \& A. Rodr.

${ }^{1}$ C. pseudomoaensis Borhidi \& O. Muñiz

C. punctata Griseb.

C. rostrata Griseb.

${ }^{1}$ C. rupicola Urb.

${ }^{1}$ C. subcapitata Urb.

${ }^{1}$ C. toaensis Borhidi

${ }^{1}$ Cameraria obovalis Alain

Campylocentrum poeppigii Rolfe

Canavalia ekmanii Urb.

${ }^{1}$ Carpodiptera cubensis subsp. ophiticola Bisse

${ }^{1}$ Casasia acunae Z. M. Fernández \& Borhidi

C. calophylla A. Rich.

${ }^{1}$ C. jacquinioides Standl.

${ }^{1} C$. nigrescens subsp. moaensis Borhidi \& O. Muñiz

${ }^{1} \mathrm{C}$. nigrescens $\mathrm{C}$. Wright ex Robinson subsp. nigrescens
Casearia aquifolia $\mathrm{C}$. Wright

${ }^{1} \mathrm{C}$. bissei J. E. Gut.

${ }^{1}$ C. crassinervis Urb.

${ }^{1}$ C. emarginata C. Wright ex Griseb.

${ }^{1}$ C. moaensis Vict.

${ }^{1}$ C. ophiticola Vict.

C. sylvestris subsp. myricoides J. E. Gut.

Cassine cubensis Borhidi

${ }^{1}$ C. nipensis Borhidi

Castela spinosa Cronquist

C. victorinii Acuña \& Roig

${ }^{1}$ Catesbaea grayi Griseb.

C. holacantha C. Wright ex Griseb.

C. nana Greenm. ex Combs.

Celastrus richardii var. elaeodendroides M. Gómez

${ }^{1}$ Cestrum buxoides Ekman \& Urb.

${ }^{1}$ C. ekmanii Urb. \& O. E. Schulz

${ }^{1}$ C. moaense Borhidi \& 0 . Muñiz

${ }^{1} C$. moquinianum Dunal.

${ }^{1}$ Ceuthocarpus involucratus Aiello

${ }^{1}$ Chaetium cubanum C. L. Hitchc.

Chaetocarpus acutifolius Borhidi

${ }^{1}$ C. cordifolius Borhidi

${ }^{1}$ C. oblongatus Borhidi

${ }^{1}$ C. parvifolius Borhidi

${ }^{1}$ Chamaecrista bucherae H. S. Irwin \& R. C. Barneby

C. cupeyalensis A. Barreto \& Yakovlev

${ }^{1}$ C. taktajanii A. Barreto \& Yakovlev

${ }^{1}$ Chaptalia crassiuscula Urb.

C. ekmanii Urb.

${ }^{1}$ C. leptophylla Urb.

C. media Urb.

${ }^{1}$ C. nipensis Urb.

C. shaferi Britton \& P. Wilson

C. stenocephala Urb.

${ }^{1}$ Cheilophyllum radicans Pennell

${ }^{1}$ C. sphaerocarpum Urb.

Chiococca cubensis Urb.

Chionanthus acunae Borhidi

C. bakeri Stearn

${ }^{1}$ C. bumelioides subsp. cubensis P. A. González

${ }^{1}$ C. moncadae Borhidi

Chione venosa var. cubensis David W. Taylor

${ }^{1}$ Ciceronia chaptalioides Urb.

Cinnamodendron cubense Urb.

Cissus wrightiana Planch.

Cleome obtusa var. gamboensis Urb.

C. procumbens subsp. wrightii R. Rankin

Clerodendrum anafense Britton \& P. Wilson

C. calcicolum Britton

C. cubense Schau. subsp. cubense

C. grandiflorum Schau.

${ }^{1}$ C. lindenianum var. camagueyense $\mathrm{C}$. Moldenke

C. lindenianum A. Rich. var. lindenianum

C. nipense Urb. var. nipense

C. nipense var. pubescens $\mathrm{C}$. Moldenke

Clethra cubensis A. Rich.

Clidemia capituliflora Cogn.

C. wrightii Griseb.

${ }^{1}$ Clusia alainii Borhidi 
${ }^{1}$ C. callosa Britton

C. grisebachiana Alain

${ }^{1}$ C. moaensis Borhidi \& O. Muñiz

${ }^{1}$ C. monocarpa Urb.

${ }^{1}$ C. munizii Borhidi

C. tetrastigma Vesque

${ }^{1}$ Coccoloba acuna R. A. Howard

C. armata Griseb.

${ }^{1}$ C. baracoensis 0 . Schmidt

C. benitensis Britton

C. caesia Ekman ex 0. Schmidt.

${ }^{1}$ C. clementis R. A. Howard

C. coriacea A. Rich.

C. cowellii Britton

C. geniculata Lindau

C. microphylla Griseb.

${ }^{1}$ C. munizii Borhidi

${ }^{1}$ C. nervosa subsp. cristalensis Borhidi

${ }^{1}$ C. nervosa Alain subsp. nervosa

${ }^{1} \mathrm{C}$. nipensis Urb.

${ }^{1}$ C. oligantha Alain

${ }^{1}$ C. pallida C. Wright ex Griseb.

${ }^{1}$ C. praestans Borhidi

C. reflexa Lindau

C. retusa Griseb.

C. rufescens $\mathrm{C}$. Wright ex Lindau

${ }^{1}$ C. shaferi Britton

${ }^{1}$ C. toaensis Alain

C. wrightii Lindau

${ }^{1}$ Coccothrinax baracoensis Borhidi \& 0 . Muñiz

C. bermudezii Léon

C. clarensis Léon subsp. clarensis

C. crinita (Griseb. \& H. Wendl. ex C. H. Wright) Becc. subsp. crinita

${ }^{1}$ C. garciana Léon

C. guantanamensis O. Muñiz \& Borhidi

C. gundlachii Léon

C. miraguama subsp. arenicola Borhidi \& 0. Muñiz

${ }^{1}$ C. miraguama subsp. havanensis Borhidi \& O. Muñiz

C. miraguama Léon subsp. miraguama

C. miraguama subsp. roseocarpa Borhidi \& 0. Muñiz

${ }^{1}$ C. nipensis Borhidi \& 0 . Muñiz

${ }^{1}$ C. orientalis 0 . Muñiz \& Borhidi

C. pauciramosa Burret

C. pseudorigida Léon

${ }^{1}$ C. rigida $\mathrm{Becc}$.

C. savannarum 0. Muñiz \& Borhidi

Colubrina cubensis subsp. ekmanii Borhidi

C. glandulosa Perkins subsp. nipensis Borhidi

Columnea tincta Griseb.

Commiphora angustata Moncada

C. glauca Moncada

Comocladia platyphylla A. Rich.

Connarus reticulatus Griseb.

Conostegia lindenii Cogn.

Copernicia baileyana Léon

C. cowellii Britton \& P. Wilson

C. curtissii Becc.

${ }^{1}$ C. fallaensis Léon

C. gigas Ekman \& Burr\&

C. glabrescens H. Wendl. ex Becc. var. glabrescens
C. hospita Mart. var. hospita

C. longiglossa Léon

C. macroglossa H. Wendl. ex Becc.

C. rigida Britton \& P. Wilson

C. $x$ burretiana 0. Muñiz \& Borhidi

${ }^{1}$ C. $x$ shaferi Dahlgren \& Glassman

C. yarey Burret var. yarey

Cordia baracoensis Urb.

${ }^{1}$ C. duartei Borhidi \& 0 . Muñiz

C. dumosa Alain

${ }^{1}$ C. erythrococca Griseb

C. grisebachii Urb.

${ }^{1}$ C. moaensis Alain

C. nipensis Urb. \& Ekman.

${ }^{1}$ C. pedunculosa Griseb.

C. sauvallei Urb.

${ }^{1}$ C. setulosa Alain

${ }^{1}$ C. shaferi Alain

${ }^{1}$ C. suffruticosa Borhidi

${ }^{1}$ C. utermarkiana Borhidi

C. valenzuelana A. Rich.

Coussarea urbaniana Standl.

${ }^{1}$ Crossopetalum cristalense Borhidi

${ }^{1}$ C. shaferi Alain

${ }^{1}$ C. ternifolium subsp. moaense Borhidi \& 0. Muñiz

${ }^{1}$ C. ternifolium Alain subsp. ternifolium

${ }^{1}$ Croton acunae Borhidi

C. alainii B. W. Van Ee \& P. E. Berry

C. bispinosus C. Wright ex Sauvalle

${ }^{1}$ C. borhidii subsp. baracoensis Borhidi \& O. Muñiz

${ }^{1}$ C. borhidii 0 . Muñiz subsp. borhidii

${ }^{1}$ C. cristalensis Urb.

${ }^{1}$ C. cycloideus Borhidi \& 0. Muñiz

${ }^{1}$ C. ekmanii Urb.

${ }^{1}$ C. holguinensis Borhidi

${ }^{1}$ C. leonis B. W. Van Ee \& P. E. Berry

${ }^{1}$ C. miraflorensis Borhidi

${ }^{1}$ C. moanus Urb.

${ }^{1}$ C. monogynus Urb.

${ }^{1}$ C. ophiticola Borhidi

${ }^{1}$ C. orientensis Borhidi

${ }^{1}$ C. pachyrachis Alain

${ }^{1}$ C. prostratus Urb.

${ }^{1}$ C. revolutus B. W. Van Ee \& P. E. Berry

C. sagraeanus Müll. Arg.

${ }^{1}$ C. trigonocarpus Griseb.

C. viminalis Griseb

Cubanola daphnoides Aiello

Cuervea integrifolia A. C. Sm.

${ }^{1}$ Cupania glabra var. multijuga Griseb.

Cuphea lobelioides Griseb.

C. parsonsia var. grisebachiana S. A. Graham

Cynanchum brachystephanum Alain

C. graminifolium Alain

${ }^{1}$ C. ophiticola Alain

C. orientense Alain

C. richardianum Alain

${ }^{1}$ Cynometra cubensis subsp. ophiticola Borhidi

Cyperus antillanus 0'neill

Dalbergaria cubensis Borhidi 
Dalechampia denticulata C. Wright ex Griseb.

${ }^{1}$ Daphnopsis angustifolia Griseb.

${ }^{1}$ D. bissei A. Noa Monzón

${ }^{1}$ D. oblongifolia Britton \& P. Wilson

${ }^{1}$ Dasytropis fragilis Urb.

${ }^{1}$ Deherainia cubensis subsp. oligospinosa Lepper

Dendrocereus nudiflorus Britton \& Rose

Dendropanax cuneifolius Seem

D. nervosus A. C. Sm.

Dendropemon claraensis Leiva

D. confertiflorus A. Leiva

${ }^{1}$ D. lepidotus subsp. cajalbanensis A. Leiva \& I. Arias

D. lepidotus A. Leiva subsp. lepidotus

${ }^{1}$ Dendrophthora confertiflora Krug \& Urb.

D. constricta Eichler.

${ }^{1}$ D. glauca Eichler. subsp. glauca

D. glauca subsp. purpurascens Leiva

${ }^{1} D$. grandifolia Eichler.

${ }^{1}$ D. lamprophylla Urb.

D. sessilifolia Krug \& Urb.

Dilomilis oligophylla Summerh.

${ }^{1}$ Dioscorea ekmanii R. Knuth.

D. nipensis R. A. Howard

D. wrightii Uline ex R. Knuth

Diospyros grisebachii Standl.

D. halesioides Griseb.

Distictis gnaphalantha Urb. subsp. gnaphalantha

Dorstenia confusa Britton

D. crenulata C. Wright

${ }^{1} D$. howardii Léon

${ }^{1}$ D. lanei R. A. Howard \& Briggs.

${ }^{1}$ D. nipensis Urb. \& Ekman

${ }^{1}$ D. tricolor Urb. \& Ekman

D. tuberosa C. Wright

Dracaena cubensis Vict.

${ }^{1}$ Drejerella tomentosula Urb.

${ }^{1}$ Drosera moaensis C. Panfet

${ }^{1}$ Echites brevipedunculatus Lippold

${ }^{1}$ E. cajalbanica Lippold

${ }^{1}$ Ekmanochloa aristata Ekman

E. subaphylla C. L. Hitchc.

Elaeodendron cubensis Bisse

Elemifera lineata Kuntze

Eleocharis oligantha C. B. Clarke

Elytraria cubana Alain

${ }^{1} E$. filicaulis Borhidi \& O. Muñiz

${ }^{1}$ E. planifolia subsp. acunae Borhidi \& O. Muñiz

${ }^{1}$ E. planifolia Leonard subsp. planifolia

E. shaferi Leonard

E. spathulifolia Borhidi \& O. Muñiz

Encyclia bipapularis Acuña

E. boothiana Dressler

${ }^{1}$ E. howardii H. Dietrich

E. oxypetala Acuña

E. phoenicea J. Neumayer

E. polybulbon subsp. cubincola Nir

E. replicata Acuña

E. triangulifera Acuña

${ }^{1}$ Eosanthe cubensis Urb.

Epidendrum hioramii Acuña
${ }^{1}$ Erigeron paucilobus Urb.

E. thrincioides Griseb.

Eriocaulon fuliginosum C. Wright

Eriochloa ekmanii C. L. Hitchc.

${ }^{1}$ E. setosa var. ekmanii R. B. Shaw

E. setosa C. L. Hitchc. var. setosa

${ }^{1}$ Erythrina acunae Borhidi var. setosa

${ }^{1}$ E. acunae Borhidi

E. cubensis C. Wright

Erythroxylum alaternifolium A. Rich. var. alaternifolium

${ }^{1}$ E. alaternifolium var. parvifolium Alain

${ }^{1}$ E. alaternifolium var. suborbiculare Alain

E. coriaceum Britton \& P. Wilson

${ }^{1}$ E. dumosum Alain

${ }^{1}$ E. flavicans Borhidi

E. havanense Rich.

${ }^{1}$ E. horridum Borhidi \& Oviedo

${ }^{1}$ E. longipes 0. E. Schulz

E. pedicellare 0. E. Schulz

E. spinescens A. Rich.

Espadaea amoena A. Rich.

Eugenia acrantha Urb.

${ }^{1}$ E. alainii Borhidi

${ }^{1}$ E. anthacanthoides Urb. \& Ekman

E. asperifolia Berg

${ }^{1}$ E. atricha Urb.

E. bayatensis Urb.

${ }^{1}$ E. beyeri Urb.

${ }^{1}$ E. cajalbanica Borhidi \& O. Muñiz

E. camarioca C. Wright

${ }^{1}$ E. canapuensis Urb.

E. cincta Griseb.

${ }^{1}$ E. cristalensis Urb.

E. cupuligera Urb.

E. cycloidea Urb.

E. cyphophloea Griseb.

E. excisa Urb.

E. farameoides A. Rich.

E. galalonensis Krug \& Urb.

E. grisebachii Krug \& Urb.

E. heterophylla A. Rich.

E. laeteviridis Urb.

${ }^{1}$ E. ligustroides Urb.

${ }^{1}$ E. lucens Alain

E. megalopetala Griseb.

${ }^{1}$ E. mensurensis Urb.

${ }^{1}$ E. mucronata Berg

E. nematopoda Urb.

${ }^{1}$ E. nipensis Urb

E. oligandra Krug \& Urb.

E. papayoensis Urb.

E. peninsularis Urb.

${ }^{1}$ E. piedraensis Urb.

E. pinetorum Urb.

E. plicatula C. Wright

${ }^{1}$ E. pocsiana Borhidi

${ }^{1}$ E. ramonae Borhidi \& 0 . Muñiz

E. ramoniana Urb.

E. rigidifolia A. Rich.

E. rigidula Britton \& P. Wilson 
E. rimosa C. Wright \& Sauvalle

E. rosariensis Borhidi

E. sauvallei Krug \& Urb.

E. scaphophylla C. Wright

E. sebastiani Urb.

${ }^{1}$ E. serrei Urb.

E. shaferi Urb.

${ }^{1}$ E. stenoptera Urb.

${ }^{1}$ E. stenoxipha Urb.

${ }^{1}$ E. stereophylla Urb.

${ }^{1}$ E. sturrockii R. A. Howard

E. subdisticha Urb.

${ }^{1}$ E. subspinulosa Borhidi \& 0. Muñiz

${ }^{1}$ E. toaensis Borhidi \& O. Muñiz

E. tuberculata DC.

${ }^{1}$ E. woodfrediana Urb.

Euphorbia brittonii Millsp.

E. camagueyensis Urb.

E. centunculoides Kunth

E. crassinodis Urb.

${ }^{1}$ E. cubensis Boiss.

${ }^{1}$ E. filicaulis Urb.

${ }^{1}$ E. helenae subsp. grandifolia Borhidi \& O. Muñiz

E. helenae Urb. subsp. helenae

E. mendezii Boiss.

${ }^{1}$ E. munizii Borhidi

${ }^{1}$ E. podocarpifolia Urb.

E. scutiformis V. W. Steinm. \& P. E Berry

${ }^{1}$ Evolvulus minimus V. Ooststr.

Exogonium argentifolium House

E. fuchsioides House

E. jalapoides House

${ }^{1}$ Exostema lancifolium Borhidi \& Acuña

E. myrtifolium Griseb. var. myrtifolium

E. parviflorum L. C. Rich. ex H. \& B

E. purpureum Griseb. subsp. purpureum

E. rotundatum Griseb.

E. stenophyllum Britton

Fagara dumosa Griseb.

Feddea cubensis Urb.

Ficus membranacea C. Wrigh

${ }^{1}$ Forchhammeria emarginata Alain

${ }^{1}$ Eurya conocarpa O.C. Schmidt

Galactia brachyodon Griseb.

G. combsii Urb.

G. earlei Britton subsp. earlei

${ }^{1}$ G. earlei subsp. toaensis Borhidi \& O. Muñiz

G. galactioides C. L. Hitchc.

G. minutifolia Urb.

${ }^{1}$ G. revoluta Urb.

G. savannarum Britton

Garcinia aristata Borhidi

${ }^{1}$ G. bakeriana Borhidi

G. clarensis Borhidi

G. cubensis Borhidi

G. moaensis Borhidi

G. ophiticola Borhidi

${ }^{1} G$. polyneura Borhidi

${ }^{1}$ G. revoluta Borhidi

G. ruscifolia Borhidi
G. serpentini Borhidi

Gesneria acuminata Urb.

G. bracteosa Urb.

G. cubensis Kuntze var. cubensis

${ }^{1} G$. depressa Urb.

G. duchartreoides C. Wright

G. ferruginea Urb.

G. libanensis Linden ex Morren subsp. libanensis

G. libanensis subsp. lopezii Borhidi

G. nipensis Britton \& P. Wilson

G. norlindii Urb.

${ }^{1}$ G. pachyclada Urb.

G. pallida Morton

G. purpurascens Urb. var. purpurascens

G. purpurascens var. yumuriensis Borhidi

G. salicifolia subsp. gibberosa Borhidi

G. salicifolia Urb. subsp. salicifolia

${ }^{1}$ G. salicifolia subsp. spathulata Borhidi

G. shaferi Urb.

G. viridiflora var. obovata Morton

G. viridiflora Kuntze subsp. viridiflora

Ginoria americana Jacq.

G. curvispina Koehne

G. ginorioides Britton

G. koehneana Urb.

${ }^{1}$ G. microphylla O. Schmidt.

Gochnatia attenuata Jervis \& Alain

G. cowellii Jervis \& Alain

${ }^{1}$ G. crassifolia Jervis \& Alain

${ }^{1}$ G. cubensis Jervis \& Alain var. cubensis

${ }^{1}$ G. cubensis var. inermis Alain

${ }^{1}$ G. elliptica subsp. inermis Alain

${ }^{1}$ G. gomezii Jervis \& Alain

${ }^{1}$ G. intertexta Jervis \& Alain

${ }^{1}$ G. maisiana Jervis \& Alain subsp. maisiana

G. obtusifolia Jervis \& Alain

${ }^{1}$ G. parvifolia Jervis \& Alain

${ }^{1}$ G. recurva Jervis \& Alain

${ }^{1}$ G. shaferi Jervis \& Alain

Gordonia moaensis H. Keng

G. wrightii H. Keng

Gouinia gracilis Ekman

Grisebachianthus hypoleucus R. M. King \& H.Rob.

G. lantanifolius R. M. King \& H.Rob.

G. libanotica R. M. King \& H. Rob.

${ }^{1}$ G. mayarensis R. M. King \& H. Rob.

${ }^{1}$ G. nipensis R. M. King \& H. Rob.

G. plucheoides R. M. King \& H. Rob.

${ }^{1}$ Guapira cajalbanensis M. A. Díaz

G. obtusata var. aberrans Alain

G. ophiticola Borhidi

${ }^{1}$ G. rufescens var. rufescens Borhidi

Guatteria cubensis Bisse

${ }^{1}$ Guettarda baracoensis Bisse

G. calcicola Britton

G. calyptrata A. Rich.

G. camagueyensis Britton

${ }^{1} G$. clarensis Britton ex Sauvalle

${ }^{1} G$. crassipes Britton

G. echinodendron C. Wright ex Sauvalle 
G. ferruginea C. Wright ex Griseb.

G. macrocarpa Griseb.

G. monocarpa Urb.

${ }^{1}$ G. retusa C. Wright ex Sauvalle

G. rigida A. Rich.

${ }^{1}$ G. roigiana Borhidi \& 0. Muñiz

G. sciaphila Urb.

G. shaferi Standl.

Guilleminea brittonii Mears var. brittonii

Gundlachia apiculata Britton \& Blake

${ }^{1}$ G. cubana Britton \& Blake

G. foliosa Britton \& Blake

Gymnanthes albicans Urb.

Gymnosiphon niveus Urb.

Habenaria bicornis Lindl.

Haenianthus grandifolius Urb.

H. variifolius Urb.

${ }^{1}$ Harnackia bisecta Urb.

${ }^{1}$ Harpalyce acunae Borhidi \& 0 . Muñiz

${ }^{1} H$. alaini Léon

${ }^{1} H$. angustiflora Léon \& Alain

${ }^{1} H$. baracoensis Borhidi \& O. Muñiz

${ }^{1} H$. borhidii O. Muñiz

${ }^{1}$ H. cristalensis Borhidi \& O. Muñiz

${ }^{1} H$. cubensis Griseb.

${ }^{1}$ H. ekmanii var. angustiflora Arroyo

${ }^{1}$ H. ekmanii Urb. var. ekmanii

${ }^{1}$ H. flexuosa Léon et Alain ex Borhidi \& 0. Muñiz

H. macrocarpa Britton \& P. Wilson

${ }^{1} H$. moana Borhidi \& 0 . Muñiz

H. nipensis Urb.

${ }^{1} H$. suberosa Urb.

${ }^{1} H$. toaensis Borhidi \& 0 . Muñiz

H. villosa Britton \& P. Wilson

Harrisia eriophora Britton

Hebestigma cubense Urb.

${ }^{1}$ Hedyosmum subintegrum Urb.

Helicteres furfuracea subsp. ophiticola A. Rodr.

${ }^{1} H$. trapezifolia A. Rich.

${ }^{1}$ Helietta cubensis Monach. \& C. Moldenke

H. glaucescens Urb.

${ }^{1}$ Hemithrinax compacta M. Gómez

${ }^{1} H$. rivularis León

${ }^{1}$ Henriettea acunae Alain

${ }^{1} H$. cuabae Borhidi

${ }^{1}$ H. squamata Alain

Heptanthus cordifolius Britton

${ }^{1} H$. lobatus Britton

H. ranunculoides Griseb.

${ }^{1} H$. shaferi Britton

${ }^{1}$ Herreranthus rivalis B. Nord

${ }^{1}$ Hottea ekmanii Borhidi

${ }^{1} H$. moana Borhidi

${ }^{1}$ Hybanthus urbanianus Melch.

H. wrightii Urb.

Hydrocotyle oligantha Urb.

Hyeronima havanensis Urb.

H. nipensis Urb.

Hylocereus cubensis Britton \& Rose

Hyperbaena angustifolia $\mathrm{Urb}$.
H. columbica Miers. subsp. columbica

H. cubensis Urb.

H. longiuscula Miers. var. longiuscula

${ }^{1}$ H. obovata Urb.

H. racemosa Urb.

Hypericum styphelioides subsp. clarense Lippold

${ }^{1}$ H. styphelioides subsp. moaense Lippold

${ }^{1}$ Hypericum styphelioides subsp. orientensis Lippold

Hyptis minutifolia Griseb.

${ }^{1}$ Ichnanthus conjunctus C. L. Hitchc. \& Ekman

I. mayarensis C. L. Hitchc.

Ilex bahiahondica P. A. González

II. baracoensis Borhidi

I. ekmaniana Loes.

1. gundlachiana Loes.

1. hypaneura Loes.

I. ligustrina Jacq.

1. manitzii P. A. Gonz-Lez

${ }^{1}$ I. moaensis Borhidi

II. moana Borhidi \& O. Muñiz

${ }^{1}$ I. victorini Alain

Illicium cubense A. C. Sm.

Ipomoea alterniflora Griseb.

I. arnoldsonii Urb.

I. cubensis Urb.

I. hypargyrea Griseb.

I. lacteola House

II. merremioides Alain

I. trinitensis Britton

Isachne leersioides Griseb.

${ }^{1}$ Isidorea ophiticola Borhidi \& Z. M. Fernández

Jacaranda arborea Urb.

J. cowellii Britton \& P. Wilson

Jacquemontia nipensis A. H. Liogier

J. serpyllifolia Urb.

Jacquinia aculeata Mez.

${ }^{1} J$. acunana Borhidi \& 0 . Muñiz

J. brunnescens Urb.

${ }^{1} J$. moana Borhidi

${ }^{1} J$. nipensis Borhidi

${ }^{1} J$. obovata Urb.

${ }^{1}$ J. oligantha Borhidi

${ }^{1} J$. robusta Urb.

J. roigii P. Wilson

${ }^{1} \mathrm{~J}$ sessiliflora Alain

J. stenophylloides Borhidi

${ }^{1}$ Jatropha minor Urb.

J. tupifolia Griseb.

Justicia sagraeana Alain

Karwinskia bicolor Urb.

K. oblongifolia Urb.

${ }^{1}$ K. orbiculata Urb.

${ }^{1}$ Koanophyllon clementis R. M. King \& H. Rob.

K. ekmanii R. M. King \& H. Rob

K. grandiceps R. M. King \& H. Rob

${ }^{1}$ K. grisebachianum R. M. King \& H. Rob

${ }^{1} K$. minutifolium R. M. King \& H. Rob

${ }^{1}$ K. oligadenium R. M. King \& H. Rob

K. polystictum R. M. King \& H. Rob

${ }^{1} K$. rhexioides R. M. King \& H. Rob. 
${ }^{1}$ Kodalyodendron cubensis Borhidi \& Acuña

${ }^{1}$ Koehneola repens Urb.

${ }^{1}$ Krokia albescens Bisse

${ }^{1}$ Lachnorhiza micrantha Borhidi

L. piloselloides A. Rich. subsp. piloselloides

Laetia ternstroemioides Griseb.

${ }^{1}$ Lagenocarpus cubensis Kük.

${ }^{1}$ Lagetta valenzuelana subsp. pauciflora A. Noa

L. valenzuelana A. Rich. subsp. valenzuelana

${ }^{1}$ Lantana camara var. rubella $\mathrm{C}$. Moldenke

Laplacea benitoensis 0. Schmidt.

${ }^{1}$ L. cristalensis Borhidi \& O. Muñiz

Lepanthes cubensis Hespenheid.

L. grisebachiana Hespenheid.

L. melanocaulon Schltr.

L. trichodactyla Lindl.

Lepidaploa gnaphaliifolia $\mathrm{H}$. Rob.

L. leptoclada H. Rob.

${ }^{1} L$. orbicularis H. Rob.

L. pineticola $\mathrm{H}$. Rob.

L. sagraeana $\mathrm{H}$. Rob.

${ }^{1}$ L. segregata $H$. Rob.

L. sericea $H$. Rob.

Leptocereus leoni Britton \& Rose

${ }^{1}$ Lescaillea equisetiformis Griseb.

${ }^{1}$ Leucocroton acunae Borhidi

${ }^{1}$ L. anomalus Borhidi

${ }^{1}$ L. bracteosus Urb.

${ }^{1}$ L. brittonii Alain

${ }^{1}$ L. comosus Urb.

L. cordifolius Alain

${ }^{1}$ L. discolor Urb.

${ }^{1}$ L. ekmanii Urb.

L. flavicans Müll. Arg.

L. havanensis Borhidi

${ }^{1}$ L. incrustatus Borhidi

${ }^{1}$ L. linearifolius Britton

${ }^{1}$ L. longibracteatus Borhidi

L. microphyllus Pax \& K. Hoffm.

L. moncadae Borhidi

${ }^{1}$ L. obovatus Urb.

${ }^{1}$ L. pachyphylloides Borhidi

${ }^{1}$ L. pachyphyllus Urb.

${ }^{1}$ L. pallidus Britton

L. revolutus $\mathrm{C}$. Wright

${ }^{1}$ L. sameki Borhidi

${ }^{1}$ L. saxicola Britton

${ }^{1}$ L. stenophyllus Urb.

${ }^{1}$ L. subpeltatus var. epeltatus Alain

${ }^{1}$ L. subpeltatus Urb. var. subpeltatus

L. virens Griseb.

Liabum wrightii Griseb.

Linodendron aronifolium Griseb.

L. cubanum Griseb.

L. venosum Griseb.

${ }^{1}$ Linum cubense Bisse

Liparis viridipurpurea Griseb.

Lisianthus glandulosus A. Rich.

L. silenifolius Urb.

${ }^{1}$ Lithachne pinetii Chase
Lobelia shaferi Urb.

Lonchocarpus blainii C. Wright ex Sauvalle

Lunania cubensis Turcz.

L. dodecandra C. Wright

${ }^{1}$ L. subcoriacea Britton \& P. Wilson

Lyonia glandulosa Griseb. var. glandulosa

${ }^{1} L$. glandulosa var. revolutifolia Judd

${ }^{1}$ L. longipes Urb.

L. macrophylla Ekman ex Urb

L. myrsinifolia Urb.

L. nipensis var. depressinerva Judd

${ }^{1}$ L. nipensis subsp. nipensis Borhidi

L. obtusa Griseb. var. obtusa

${ }^{1}$ Machaerina cubensis T. Koyama

${ }^{1} M$. filifolia Griseb.

${ }^{1}$ Machaonia dumosa Borhidi \& Z. M. Fernández

M. havanensis Alain subsp. havanensis

M. micrantha Borhidi \& Z. M. Fernández

M. microphylla Griseb.

${ }^{1}$ M. nipensis subsp. moaensis Borhidi \& Z. M. Fernández

M. nipensis Borhidi \& Z. M. Fernández subsp. nipensis

M. pauciflora subsp. trifurcata Borhidi \& Z. M. Fernández

M. subinermis subsp. armata Borhidi \& Z. M. Fernández

M. subinermis Urb. subsp. subinermis

${ }^{1}$ M. tiffina Urb. \& Ekman

${ }^{1}$ M. urbinoi Borhidi \& O. Muñiz

${ }^{1}$ Macrocarpaea pinetorum Alain

Magnolia cristalensis Bisse

Magnolia cubensis Urb. subsp. cubensis

M. minor R. Govaerts

${ }^{1}$ Malpighia apiculata Urb.

${ }^{1}$ M. cristalensis F. K. Meyer subsp. cristalensis

M. cubensis Kunth

${ }^{1}$ M. martiana Acuña \& Roig

${ }^{1}$ M. nummulariifolia subsp. arroyensis F. K. Meyer

M. nummulariifolia subsp. camagueyensis F. K. Meyer

${ }^{1} M$. nummulariifolia subsp. clarensis F. K. Meyer

${ }^{1}$ M. nummulariifolia subsp. holguinensis F. K. Meyer

M. nummulariifolia Nied. subsp. nummulariifolia

${ }^{1} M$. setosa subsp. moaensis Meyer

M. suberosa Small

Manilkara jaimiqui Dubard subsp. jaimiqui

M. jaimiqui subsp. wrightiana Cronquist

M. mayarensis Cronquist subsp. mayarensis

Manisuris impressa Kuntze

Marcgravia evenia Krug \& Urb.

Margaritaria tetracocca G. L. Webster

Marila dissitiflora C. Wright

${ }^{1}$ Marsdenia cubensis Turcz.

${ }^{1}$ M. micrantha Alain

M. umbellata Griseb.

${ }^{1}$ Matelea acuminata R. E. Woodson

M. bayatensis R. E. Woodson

M. nipensis R. E. Woodson

M. oblongata R. E. Woodson

M. ovatifolia R. E. Woodson

${ }^{1}$ M. tamnifolia R. E. Woodson

${ }^{1}$ Maytenus cajalbanica Borhidi

M. loeseneri Urb.

M. revoluta Alain 
${ }^{1}$ M. serpentini Borhidi

Mazaea phialanthoides Krug \& Urb.

${ }^{1}$ M. shaferi Delprete

Mecranium haemanthum Triana

${ }^{1}$ M. obtusifolium Cogn.

Melocactus matanzanus Léon

${ }^{1}$ Melochia nipensis Britton

Mesechites minima Wodson

M. rosea Miers.

Mesosetum wrightii C. L. Hitchc.

Metopium venosum Engler

Miconia alternifolia Alain

M. baracoensis Urb.

${ }^{1} M$. bucherae Alain

${ }^{1}$ M. cerasiflora Urb. var. cerasiflora

${ }^{1} M$. cerasiflora var. setulifera Urb.

${ }^{1} M$. javorkaeana Borhidi

M. lenticellata Alain

M. moensis Alain

${ }^{1}$ M. petersonii Urb.

${ }^{1} M$. rhombifolia Alain

${ }^{1} M$. shaferi Cogn.

${ }^{1}$ M. uninervis Alain

${ }^{1} M$. victorini Alain

${ }^{1}$ Micrasepalum eritrichoides Urb.

Micropholis polita Pierre

Mikania alba N. Taylor

M. lindenii S. Moore

M. ranunculifolia A. Rich.

Mimosa ekmanii Urb.

M. fagaracantha Griseb.

${ }^{1}$ M. moaensis Britton \& P. Wilson

Misanteca cubensis Benth.

Mitracarpus acunae Alain

${ }^{1}$ M. squarrosus DC.

Mitranthes ottonis Berg

Mniochloa pulchella Chase

M. strephioides Chase

${ }^{1}$ Moacroton gynopetalus Borhidi

${ }^{1}$ M. tetramerus Borhidi \& 0. Muñiz

Mollugo cuneifolia Urb.

${ }^{1} M$. deltoidea Léon

Morella punctata J. Herb.

${ }^{1}$ Morinda moaensis Alain

Mosiera acunae Borhidi

M. bullata Bisse

${ }^{1}$ M. cabanasensis subsp. flavicans Borhidi

M. calycolpoides Borhidi

${ }^{1}$ M. del-riscoi Borhidi

M. ekmanii Bisse

${ }^{1} M$. moaense Bisse

${ }^{1}$ M. moaensis Bisse

M. munizii Bisse

M. oonophylla Bisse

${ }^{1}$ M. ophiticola Bisse

Mouriri emarginata Griseb var. emarginata

M. emarginata var. rostrata Morley

M. myrtilloides subsp. acuta Morley

M. spathulata Griseb var. spathulata

M. valenzuelana A. Rich.
${ }^{1}$ Myrcia acunae Borhidi

${ }^{1} M$. albescens Alain

${ }^{1}$ M. apodocarpa Urb.

${ }^{1} M$. borhidii 0 . Muñiz

${ }^{1}$ M. cristalensis Borhidi \& O. Muñiz

${ }^{1} M$. pineticola Borhidi \& 0 . Muñiz

M. retivenia Urb.

${ }^{1}$ M. spinifolia Borhidi \& 0. Muñiz

${ }^{1} M$. susannae Borhidi

${ }^{1} M$. toaensis Borhidi \& 0. Muñiz

Myrciaria floribunda Spreng.

M. formosa Berg

${ }^{1}$ Myrica shaferi Urb. \& Britton

${ }^{1}$ Myrsine cristalensis Borhidi

Myrtus micarensis Urb.

M. navasense Bisse

${ }^{1} M$. reversa Bisse

Nashia myrtifolia C. Moldenke

N. nipensis C. Moldenke

Nectandra minima J. G. Rohwer

Neobesseya cubensis Hester

${ }^{1}$ Neobracea acunaiana Lippold

${ }^{1}$ N. ekmanii Urb.

$N$. valenzuelana Urb.

Neomezia cubensis Votsch. subsp. cubensis

${ }^{1}$ Nodocarpaea radicans A. Gray

Notodon cayensis Britton \& P. Wilson

${ }^{1} N$. savannarum Britton \& P. Wilson

${ }^{1}$ Ocotea baracoensis Borhidi \& Imchanitskaya

${ }^{1}$ O. bucherii Roig \& Acuña

${ }^{1}$ O. cristalensis Bisse

O. moaensis Bisse

Octomeria prostrata $\mathrm{H}$. Stenzel

${ }^{1}$ O. ventii H. Dietrich

Oldenlandia capillipes Griseb.

${ }^{1}$ O. polyphylla Urb.

Oldfeltia polyphlebia B. Nord. \& Lundin

Oplonia cubensis Borhidi

O. moana Borhidi

${ }^{1}$ O. multigemma Borhidi

O. nannophylla Stearn

O. polyece Borhidi

O. tetrasticha Stearn sensu Borhidi subsp. tetrasticha

${ }^{1}$ Ossaea acunae Alain

${ }^{1} O$. anomala Borhidi

${ }^{1}$ O. baracoensis Borhidi \& O. Muñiz

O. brunescens Urb.

O. ekmanii Urb.

${ }^{1}$ O. elliptica Alain

${ }^{1}$ O. granulata Urb.

${ }^{1}$ O. hypoglauca Benth. \& Hook. f.

O. involucrata C. Wright ex Sauvalle

O. lanata C. Wright ex Griseb.

1O. micarensis Urb.

10. moaensis Alain

O. muricata C. Wright ex Sauvalle

${ }^{1}$ O. navasensis Britton \& P. Wilson

O. neurotricha C. Wright ex Sauvalle

${ }^{1}$ O. nipensis Britton \& P. Wilson

O. ovatifolia Urb. 
O. pauciflora Griseb.

${ }^{1}$. pilifera Urb.

${ }^{1}$ O. pinetorum Alain

${ }^{1}$ O. pulchra Alain

O. rufescens C. Wright ex Sauvalle

${ }^{1}$ O. shaferi Britton \& P. Wilson

${ }^{1} O$. trianaei Cogn.

${ }^{1}$ O. vazquezii Borhidi \& 0 . Muñiz

${ }^{1} O$. verrucosa $\mathrm{M}$. Gómez

${ }^{1}$ Ouratea alternifolia M. Gómez

O. elliptica M. Gómez

O. ilicifolia var. savannarum Dwyer

O. revoluta Engler

${ }^{1}$ Oxalis cajalbanensis Urb.

Pachira cubensis Fern. Alonso

${ }^{1}$ Pachyanthus discolor Norlind.

${ }^{1} P$. mayarensis Urb.

${ }^{1}$ P. moaensis Borhidi

${ }^{1}$ P. monocephalus Borhidi

${ }^{1} P$. neglectus subsp. baracoensis Borhidi

${ }^{1} P$. neglectus Borhidi subsp. neglectus

${ }^{1}$. oleifolius Griseb.

P. pedicellatus Urb.

P. poiretii Griseb.

P. reticulatus Britton \& P. Wilson

${ }^{1}$ Paepalanthus nipensis Gonz. Geigel

'P. pungens Griseb.

${ }^{1}$ P. riparius $\mathrm{C}$. Moldenke

${ }^{1}$ Panicum beyeri C. L. Hitchc. \& Ekman

${ }^{1}$ Paspalidium subtransiens Davidse \& R. W. Pohl

Paspalum acutifolium Léon

P. capillifolium Nash

1P. edmondi Léon

${ }^{1}$. motembense Léon

$P$. rocanum Léon

Passiflora cubensis Urb.

${ }^{1}$. nipensis Britton

P. shaferi Britton

'P. stenoloba Urb.

Pectis cubensis Griseb.

${ }^{1}$ P. havanensis Urb.

${ }^{1}$ Pentacalia almironcillo Proctor

Peperomia cubensis C. DC.

P. cueroensis Britton

P. pseudo-pereskiaefoli C. DC.

'P. subrotundifolia C. DC.

Pera ekmanii Urb.

${ }^{1}$ P. longipes Britton \& P. Wilson

P. orientensis Borhidi

${ }^{1}$ P. ovalifolia Urb.

${ }^{1}$ P. polylepis subsp. moaensis Borhidi

${ }^{1}$ P. polylepis Urb. subsp. polylepis

'Phania cajalbanica Borhidi \& Muñiz

Phenax asper Wedd.

'Phialanthus acunae Borhidi

1P. ellipticus Urb.

'P. glaberrimus Borhidi

${ }^{1} P$. inflatus Borhidi

${ }^{1} P$. linearis Alain

${ }^{1}$ P. macrocalyx Borhidi
1. macrostemon Standl.

P. oblongatus Urb.

${ }^{1}$ P. parvifolius Urb.

${ }^{1}$ P. resinifluus Griseb.

P. rigidus subsp. pinosius Borhidi

P. rigidus Griseb. subsp. rigidus

Phidiasia lindavii Urb.

Philodendron scandens subsp. cubense Arias

Phinaea pulchella Morton

Phoradendron gundlachii Krug \& Urb.

Phyllanthus carnosulus Müll. Arg.

P. chamaecristoides subsp. baracoensis G. L. Webster

${ }^{1} P$. chamaecristoides Urb. subsp. chamaecristoides

${ }^{1}$ P. chryseus R. A. Howard

${ }^{1} P$. comosus Urb.

${ }^{1} P$. comptus G. L. Webster

${ }^{1}$. cristalensis Urb.

P. discolor Spreng.

${ }^{1}$ P. ekmanii G. L. Webster

P. epiphyllanthus subsp. dilatatus G. L. Webster

P. excisus Urb.

${ }^{1}$ P. formosus Urb.

${ }^{1}$ P. incrustatus Urb.

P. junceus Müll. Arg.

P. maestrensis Urb.

${ }^{1}$ P. microdictyus Urb.

${ }^{1}$ P. mirificus G. L. Webster

${ }^{1}$ P. myrtilloides subsp. alainii $\mathrm{G}$. L. Webster

P. myrtilloides subsp. erythrinus G. L. Webster

P. myrtilloides Griseb. subsp. myrtilloides

1P. myrtilloides subsp. shaferi G. L. Webster

${ }^{1}$ P. myrtilloides subsp. spathulifolius G. L. Webster

${ }^{1}$ P. norlindii Urb.

P. orbicularis Kunth

P. pachystylus Urb.

${ }^{1}$ P. pallidus C. Wright ex Griseb.

${ }^{1}$ P. phlebocarpus Urb.

P. procerus C. Wright ex Sauvalle

${ }^{1}$ P. pseudocicca Griseb.

1P. scopulorum Urb.

P. subcarnosus C. Wright ex Müll. Arg.

${ }^{1} P$. williamioides Griseb.

P. x pallidus C. Wright ex Griseb.

Phyllomelia coronata Griseb.

Picardaea cubensis Britton ex Urb.

Picramnia reticulata Griseb.

Pictetia angustifolia Griseb.

P. cubensis Bisse

P. mucronata Beyra \& Lavin

${ }^{1}$ P. nipensis Beyra \& Lavin

Pilea affinis Morton var. affinis

${ }^{1}$ P. ambecarpa Urb.

P. bullata Britton

P. cubensis Wedd.

P. ermitensis Britton

${ }^{1}$ P. floridana Urb.

P. forsythiana var. robustior Wedd.

P. fruticulosa Morton

$P$. heteroneura Griseb.

P. intermedia Urb. 
${ }^{1}$ P. mayarensis Morton

${ }^{1}$ P. nipensis Urb.

P. nudicaulis Wedd

${ }^{1}$ P. ophiticola Borhidi

${ }^{1}$ P. orientalis Morton

P. parciflora Urb.

P. pulchra Morton

P. valenzuelae Urb.

Pilosocereus robinii Byles \& Rowley

Pimenta adenoclada Burret

P. cainitoides Burret

P. filipes Burret

${ }^{1}$ P. podocarpoides Landrum

Pinguicula albida C. Wright ex Griseb.

${ }^{1}$ P. benedicta Barnh.

${ }^{1}$ P. lignicola Barnh.

Pinus caribaea Morelet var. caribaea

P. cubensis Griseb.

Piper arboreum subsp. stamineum Borhidi

P. clementis Léon

${ }^{1}$ P. cubense C. DC.

P. holguinianum Trel.

P. mananthum C. Wright

P. ossanum Trel.

P. richardianum C. DC.

P. rigidum C. DC.

P. sphaerocarpum C. Wright

P. subpuberulum Trel.

P. yaranum Trel.

Piscidia cubensis Urb.

1Pisonia petiolaris Heimerl \& Ekman

Pitcairnia cubensis L. B. Smith

Platygyna dentata Alain

P. hexandra Müll. Arg.

P. leonis Alain

P. obovata Borhidi

P. parvifolia Alain

'P. triandra Borhidi

${ }^{1}$ P. volubilis R. A. Howard

${ }^{1}$ Platystele hyalina $\mathrm{H}$. Stenzel

Pleurothallis bovilabia C. Schweinf.

${ }^{1}$ P. ekmanii Schltr.

P. excentrica G. M. Luer

$P$. hymenantha Lindl.

P. murex Rchb. F.

'Plinia baracoensis Borhidi

P. cubensis Urb.

${ }^{1}$ P. dermatodes Urb.

${ }^{1}$ P. moaensis Borhidi

1P. punctata Urb.

P. ramosissima Urb.

1P. stenophylla Urb.

Plumeria filifolia Griseb.

Podocarpus angustifolius Griseb.

P. ekmanii Urb.

Poitea gracilis Lavin

${ }^{1}$ P. immarginata Lavin

'Polygala amara subsp. brachyptera Hayek

P. ambigens Blake

P. cuneata Blake
P. montana Blake

P. rhynchosperma Blake

P. saginoides Griseb.

P. scabridula Blake

P. stipitata Blake

P. wrightii Chod.

Polystachya concreta Garay \& H. R. Sweet

${ }^{1}$ Portulaca teretifolia var. cubensis Legrand

Pouteria cubensis Baehni

P. dictyoneura Radkl. subsp. dictyoneura

${ }^{1}$ P. micrantha Baehni

Prosthechea ochrantha J.M.H.Shaw

${ }^{1}$ Protium baracoense Bisse

P. cubense Urb.

${ }^{1}$ P. fragrans Urb.

P. subacuminatum $\mathrm{Sw}$.

Pseudocarpidium neglecta Bisse

P. pungens Britton

${ }^{1}$ P. rigens Britton

Pseudosamanea cubana R. C. Barneby \& J. W. Grimes

Psidium balium Urb.

P. celastroides Urb.

${ }^{1}$ P. cymosum Urb.

${ }^{1}$ P. jackucsianum Borhidi

P. minutifolium Krug \& Urb.

P. parvifolium Griseb.

P. tenuirame Urb.

${ }^{1}$ Psychotria agustinae Acuña

1P. alaini Acuña \& Roig

P. auriculata C. Wright ex Griseb.

${ }^{1}$ P. bermejalensis Britton

P. bialata C. Wright ex Griseb.

${ }^{1}$ P. byrsonimaefolia Acuña \& Roig

P. cathetoneura Urb.

P. clementis Britton

P. costivenia Griseb.

${ }^{1}$ P. cristalensis Urb.

${ }^{1}$ P. evenia C. Wright ex Griseb.

P. graminifolia Urb.

P. lasiophthalma Griseb.

P. leonis Britton

${ }^{1}$ P. lopezii Acuña \& Roig

${ }^{1}$ P. moensis Britton \& P. Wilson

1P. moralesi Acuña \& Roig

P. odorata C. Wright ex Griseb.

P. ossaeana Urb.

P. pachythala Urb.

${ }^{1}$ P. ponce-leonis Acuña \& Roig

P. rectinervis Urb.

${ }^{1}$ P. rufovaginata Griseb.

${ }^{1} P$. shaferi Urb.

${ }^{1}$ P. subalata Ws. ex Griseb.

${ }^{1}$ P. thelophora Urb.

P. toensis Britton \& P. Wilson

P. torrei Acuña \& Roig

P. van-hermannii Acuña \& Roig

1 Purdiaea ekmanii Vict.

1P. microphylla Britton \& P. Wilson

${ }^{1}$ P. moaensis Vict.

P. nipensis Vict. subsp. nipensis 
1P. shaferi Britton \& P. Wilson

${ }^{1}$ P. stereosepala J. L. Thomas

P. velutina Britton \& P. Wilson

Rajania cephalocarpa Uline ex R. Knuth

R. microphylla Kunth.

R. nipensis R. A. Howard

${ }^{1} R$. porulosa $\mathrm{R}$. Knuth

${ }^{1} R$. tenella R. A. Howard

R. tenuiflora R. Knuth

Randia cubana Borhidi

Rauvolfia cubana A. DC.

${ }^{1}$ R. linearifolia Britton \& P. Wilson

R. salicifolia Griseb.

${ }^{1}$ Ravenia baracoensis Borhidi

${ }^{1} R$. carabiai Vict.

${ }^{1}$ R. ekmanii P. Wilson

R. leonis Vict.

R. simplicifolia C. Wright ex P. Wilson

${ }^{1}$ Reynosia latifolia Griseb.

${ }^{1}$ R. microphylla Urb.

${ }^{1}$ R. moaensis Borhidi \& O. Muñiz

${ }^{1}$ R. mucronata subsp. nipensis Borhidi \& 0. Muñiz

R. retusa Griseb.

R. wrightii Urb.

${ }^{1}$ Rhamnidium brevifolium Borhidi

R. ellipticum Britton \& P. Wilson

${ }^{1} R$. nipense Urb.

${ }^{1} R$. pruinosum $\mathrm{Urb}$.

${ }^{1} R$. shaferi Britton \& P. Wilson

${ }^{1}$ Rhodogeron coronopifolius Griseb.

${ }^{1}$ Rhynchosia nipensis Urb.

${ }^{1}$ Rhynchospora bucherorum Léon

R. cernua Griseb. var. cernua

R. gageri Britton

${ }^{1} R$. lindeniana Griseb.

${ }^{1} R$. mayarensis Léon

R. seslerioides Griseb.

${ }^{1}$ R. shaferi Britton

${ }^{1}$ R. tenuifolia Griseb.

Rhytidophyllum coccineum Urb.

R. exsertum Griseb. subsp. exsertum

R. exsertum subsp. wrightianum Borhidi

R. tomentosum subsp. villosulum Borhidi

Rochefortia cubensis Britton \& P. Wilson

${ }^{1}$ R. oblanceata G. Klotz

${ }^{1}$ R. septentrionalis $\mathrm{G}$. Klotz

${ }^{1}$ Rondeletia acunae Borhidi \& Z. M. Fernández

$R$. alaternoides A. Rich. subsp. alaternoides

R. alaternoides subsp. brachyloba Z. M. Fernández \& Borhidi

$R$. alaternoides subsp. myrtacea Z. M. Fernández

R. baracoensis Britton

${ }^{1}$ R. combsioides Z. M. Fernández \& Borhidi

${ }^{1} R$. coronata Urb.

${ }^{1}$ R. cristalensis Urb.

${ }^{1}$ R. galanensis Z. M. Fernández \& Borhidi

${ }^{1}$ R. glomeruliflora Alain

$R$. grandisepala Alain

R. lindeniana A. Rich.

${ }^{1} R$. lomensis Urb.

${ }^{1}$ R. longibracteata Alain
${ }^{1}$ R. lucida Z. M. Fernández \& Borhidi

${ }^{1}$ R. micarensis Urb.

${ }^{1}$ R. miraflorensis Z. M. Fernández \& Borhidi

${ }^{1} R$. moaensis Alain

${ }^{1} R$. nipensis Urb.

${ }^{1}$ R. odorata subsp. bullata Z. M. Fernández \& P. Herrera

$R$. odorata Jacq. subsp. odorata

R. pachyphylla subsp. myrtilloides Z. M. Fernández \& Borhidi

R. pachyphylla Krug \& Urb. subsp. pachyphylla

R. paucinervis Urb.

R. peninsularis Z. M. Fernández \& Borhidi

${ }^{1}$ R. plicatula Urb.

${ }^{1}$ R. pycnophylla Urb.

${ }^{1} R$. shaferi Urb. \& Britton

${ }^{1}$ R. steirophylla Urb.

${ }^{1} R$. subcanescens Z. M. Fernández \& Borhidi

${ }^{1}$ R. toaensis Z. M. Fernández \& Borhidi

${ }^{1}$ R. vaccinifolia Britton

$R$. venosa C. Wright ex Griseb.

Roystonea lenis Léon

Rubus grisebachii Focke

${ }^{1}$ Sachsia tricephala Griseb.

${ }^{1}$ Salacia nipensis Britton

S. wrightii Urb.

Salmea insipida Bolick \& R. K. Jansen

Salvia cubensis Britton

S. nigrescens Alain

${ }^{1}$ S. scabrata Britton \& P. Wilson

${ }^{1}$ S. speirematoides C. Wright ex Sauvalle

Samyda macrantha P. Wilson

S. ramosissima J. E. Gut.

Sapium adenodon Griseb.

${ }^{1}$ Sapphoa ekmanii Borhidi

${ }^{1}$ S. rigidifolia Urb.

${ }^{1}$ Savia clementis Alain

S. clusiifolia Griseb.

${ }^{1}$ S. cuneifolia Urb.

S. erythroxyloides var. parvifolia Urb.

S. laurifolia Griseb.

${ }^{1}$ Schizachyrium multinervosum Nash

${ }^{1}$ Schmidtottia corymbosa Borhidi

${ }^{1}$ S. cubensis Urb.

${ }^{1}$ S. cucullata Borhidi \& Bisse

${ }^{1}$ S. elliptica Urb. subsp. elliptica

${ }^{1}$ S. elliptica subsp. oblongata Borhidi

${ }^{1} S$. marmorata Urb.

${ }^{1}$ S. monantha Urb.

${ }^{1} S$. monticola Borhidi

${ }^{1}$ S. multiflora Urb.

${ }^{1}$ S. nitens Urb.

${ }^{1}$ S. parvifolia Alain

${ }^{1} S$. scabra Borhidi \& 0. Muñiz

${ }^{1}$ S. sessilifolia Urb. subsp. sessilifolia

${ }^{1} S$. shaferi subsp. micarensis Borhidi

${ }^{1}$ S. shaferi subsp. neglecta Borhidi

${ }^{1} S$. shaferi Urb. subsp. shaferi

${ }^{1}$ S. stricta Borhidi

${ }^{1}$ S. uliginosa Urb.

${ }^{1}$ Schoepfia cubensis Britton \& P. Wilson

${ }^{1} S$. paradoxa Berazaín 
${ }^{1}$ S. scopulorum Alain

Schwenckia filiformis Ekman ex Urb.

Scleria havanensis Britton

S. lacustris C. Wright

${ }^{1}$ S. motemboensis Britton

${ }^{1}$ S. pilosissima Britton

S. stereorrhiza C. Wright

S. wrightiana Boeck.

${ }^{1}$ Scolosanthus acunae Borhidi \& O. Muñiz

S. lucidus Britton

S. maestrensis Alain

${ }^{1}$ S. moanus Borhidi \& 0. Muñiz

${ }^{1}$ S. nipensis Borhidi \& 0 . Muñiz

${ }^{1}$ S. pycnophyllus Borhidi

${ }^{1} S$. reticulatus Borhidi

Scutachne amphistemon C. L. Hitchc. \& Chase

Securidaca elliptica Turcz.

${ }^{1}$ Senecio eriocarphus Greenm.

${ }^{1} S$. leucolepis Greenm.

Senna benitoensis H. S. Irwin \& R. C. Barneby

S. chrysocarpa H. S. Irwin \& R. C. Barneby

${ }^{1}$ S. gundlachii H. S. Irwin \& R. C. Barneby subsp. gundlachii

S. shaferi A. Barreto \& Yakovlev

Serjania simulata Britton \& P. Wilson

Shafera platyphylla Greenm.

${ }^{1}$ Shaferocharis cubensis Urb.

${ }^{1}$ S. multiflora Borhidi \& 0. Muñiz

${ }^{1}$ S. villosa Borhidi \& Bisse

Sideroxylon acunae T. D. Penn.

S. jubilla T. D. Penn.

${ }^{1} S$. moaense J. E. Gut.

Simarouba laevis Griseb.

Siphocampylus baracoensis Vict.

S. cernuus Griseb. var. cernuus

${ }^{1} S$. glaber Mc Vaugh

S. manettiaeflorus Hook.

${ }^{1}$ S. patens Griseb.

S. ruber Alain

S. subglaber Urb.

S. yumuriensis Vict.

Sloanea curatellifolia Griseb.

Solanum cristalense Amshoff

S. gundlachii Urb subsp. gundlachii

${ }^{1} S$. moense Britton \& P. Wilson

${ }^{1}$ S. pachyneuroides Amshoff

S. pachyneurum O. E. Schulz

${ }^{1}$ Spachea martiana Acuña \& Roig

Spaniopappus hygrophilus R. M. King \& H.Rob.

Spaniopappus iodostylus R. M. King \& H.Rob.

Spathelia cubensis P. Wilson

S. leonis Vict.

${ }^{1}$ S. lobulata Urb.

${ }^{1}$ S. pinetorum subsp. megaphylla Vict.

${ }^{1}$ S. pinetorum Vict. subsp. pinetorum

${ }^{1}$ S. splendens Urb.

${ }^{1}$ S. stipitata Urb.

${ }^{1} S$. subintegra Vict.

S. vernicosa Planch

${ }^{1} S$. wrightii Vict.

S. yumuriensis Vict.
Specklinia grisebachiana G. M. Luer

S. lichenicola Pridgeon \& M. W. Chase

S. llamachoi G. M. Luer

S. trichyphis G. M. Luer

Spermacoce thymocephala C. Wright

Sphinga prehensilis R. C. Barneby \& J. W. Grimes

Spirotecoma apiculata Alain

S. holguinensis Alain

S. spiralis Pichon

Stelis ekmanii Schltr.

Stenandrium crenatum Urb.

S. ekmanii Urb.

${ }^{1}$ S. heterotrichum Borhidi

S. ovatum Urb.

S. wrightii Lindau

${ }^{1}$ Stenostomum abbreviatum Borhidi \& Z. M. Fernández subsp. abbreviatum

${ }^{1}$ S. abbreviatum subsp. moaensis Borhidi \& Z. M. Fernández

${ }^{1}$ S. abbreviatum subsp. obcordatum Borhidi \& Z. M. Fernández

S. aristatum Britton

${ }^{1}$ S. minutifolium Borhidi

S. mucronatum Borhidi \& Z. M. Fernández

${ }^{1}$ S. multinerve Borhidi \& Z. M. Fernández

${ }^{1}$ S. nipense Z. M. Fernández

${ }^{1}$ S. ophiticolum Borhidi \& M. Z. Fernández

${ }^{1}$ S. orbiculare Z. M. Fernández

${ }^{1}$ S. pedicellare Borhidi

${ }^{1}$ S. scrobiculatum Borhidi \& Z. M. Fernández

S. shaferi Borhidi \& Z. M. FernándeZ

S. urbanianum Borhidi

Stigmaphyllon microphyllum Griseb.

${ }^{1}$ Suberanthus canellifolius Borhidi \& Z. M. Fernández

S. neriifolius Borhidi \& Z. M. Fernández

S. stellatus Borhidi \& Z. M. Fernández

Symphysia alainii Berazaín

Symplocos leonis Britton \& P. Wilson

${ }^{1} S$. moaensis Borhidi

S. salicifolia Griseb.

S. strigillosa Krug \& Urb.

Synapsis ilicifolia Griseb.

Tabebuia arimaoensis Britton

T. bibracteolata Britton

T. brooksiana Britton

${ }^{1}$ T. clementis Alain

T. dubia Britton \& Seibert

${ }^{1}$ T. elegans Urb.

T. glaucescens Urb.

T. gracilipes Alain

T. inaequipes Urb.

T. lepidophylla Greenm.

T. leptoneura Urb.

${ }^{1}$ T. linearis Alain

T. moaensis Britton

${ }^{1}$ T. pinetorum Britton

T. pulverulenta Urb.

T. sauvallei Britton

T. shaferi Britton

T. simplicifolia Carabia ex Alain

T. trachycarpa K. Schum.

Tafallaea grisebachii Kuntze 
Tapura cubensis Griseb. subsp. cubensis

T. cubensis subsp. obovata Borhidi

${ }^{1}$ T. orbicularis Ekman ex Urb.

Terminalia chicharronia subsp. neglecta Alwan \& Stace

T. chicharronia subsp. orientensis Alwan \& Stace

T. eriostachya A. Rich.

${ }^{1}$ Ternstroemia flavescens Griseb.

${ }^{1}$ T. moaensis Borhidi \& 0. Muñiz

T. parviflora Krug \& Urb.

${ }^{1}$ Tetralix brachypetalus Griseb.

${ }^{1}$ T. cristalensis Bisse

${ }^{1}$ T. jaucoensis Bisse

${ }^{1}$ T. moaensis Bisse

${ }^{1}$ T. nipensis Urb.

Tetramicra eulophiae Rchb. F.

T. simplex Ames

${ }^{1}$ Tetrazygia barbata Borhidi

T. coriacea Urb.

${ }^{1}$ T. cristalensis Borhidi

T. elegans Urb.

Tetrazygiopsis laxiflora Borhidi

Thouinia acunae Borhidi \& 0. Muñiz

T. patentinervis Radlk.

T. pseudopunctata Lippold

T. punctata Radlk.

T. reticulata Alain

Thrinax radiata Lodd. ex Schult. \& Schult.f.

${ }^{1}$ Thymopsis thymoides var. glabrescens Alain

${ }^{1}$ T. thymoides var. polyantha Alain

Tocoyena cubensis Britton ex Standl.

${ }^{1}$ Tolumnia usneoides Braem

${ }^{1}$ Torralbasia cuneifolia subsp. verrucosa Borhidi

${ }^{1}$ Tragia cubensis Urb.

Trichilia trachyantha C. DC.

Triscenia ovina Griseb.

Vaccinium alainii Acuña \& Roig

V. cubense Griseb. var. cubense

V. ramonii Griseb.

${ }^{1} V$. shaferi Acuña \& Roig

${ }^{1}$ Varronia acunae C. Moldenke

${ }^{1} V$. holguinensis Borhidi
V. toaensis Borhidi

${ }^{1}$ Verbesina wrightii Griseb.

Vernonanthura havanensis (DC.) H. Rob.

V. hieracioides (Griseb.) H. Rob.

V. menthaefolia (Poepp. ex Spreng.) H. Rob.

Vernonia acunae Alain

V. calida Gleason

V. calophylla Gleason

${ }^{1}$ V. cristalensis Alain

${ }^{1}$. desiliens Gleason

V. inaequiserrata Sch. Bip.

${ }^{1}$ V. moaensis Alain

V. sagraeana DC.

${ }^{1}$ V. valenzuelana A. Rich.

${ }^{1}$ V. vicina Gleason

V. wrightii Sch. Bip.

Votomita monantha Morley

${ }^{1}$ Vriesea haplostachya L. B. Smith

V. platynema var. wrightii L. B. Smith

Wallenia bumelioides (Griseb.) Mez

W. jacquinioides (Griseb.) Mez

${ }^{1}$ Waltheria ovalifolia Urb.

Wedelia ehrenbergii var. veronicifolia 0 . E. Schulz

W. rugosa Greenm.

${ }^{1}$ W. serrata var. multidentata O. E. Schulz.

Xylopia ekmanii R. E. Fr.

X. obtusifolia A. Rich.

$X$. roigii P. Wilson

Xylosma acunae Borhidi \& O. Muñiz

X. buxifolium A. Gray var. buxifolium

${ }^{1} X$. iberiensis J. E. Gut.

$X$ infesta Griseb.

Xyris navicularis Griseb.

Zamia pygmaea Sims

${ }^{1}$ Zanthoxylum lomincola Alain

Z. nannophyllum Alain

Z. taediosum A. Rich.

Zephyranthes rosea Lindl.

Ziziphus acutifolia M. C. Johnst.

Z. bidens M. C. Johnst. 